\title{
Clinical phenotypes of Chinese primary hyperparathyroidism patients are associated with the calcium-sensing receptor gene R990G polymorphism
}

\author{
Guiyan Han ${ }^{* \dagger}$, Ou Wang*, Min Nie, Yan Zhu, Xunwu Meng, Yingying Hu, Huaicheng Liu and Xiaoping Xing \\ Key Laboratory of Endocrinology of Ministry of Health, Department of Endocrinology, Peking Union Medical College, Chinese Academy of Medical Sciences, \\ Peking Union Medical College Hospital, Beijing 100730, China \\ (Correspondence should be addressed to X Xing; Email: xiaopingxing@126.com)
}

*(G Han and O Wang contributed equally to this work)

${ }^{\dagger}$ (G Han is now at Department of Endocrinology, Teaching Hospital of Capital Medical University of Beijing Electric Power Hospital, Beijing 100073, China)

\begin{abstract}
Objective: The purpose of this study was to investigate the distribution of the A986S and R990G polymorphisms of the calcium-sensing receptor (CASR) gene in the Chinese population and whether there is an association between genetic variants and the risk of developing primary hyperparathyroidism (PHPT) and its associated clinical phenotypes.

Methods: A total of 164 Chinese Han PHPT patients (M/F: 51/113) and 230 healthy controls (M/F: 50/180) were enrolled. The common clinical parameters of PHPT patients including biochemical markers, bone mineral density (BMD), kidney stone occurrence, and pathology results were analyzed. Genotyping was conducted for both the patients and controls, and it was carried out using standard procedures.

Results: The R990G variant was more frequently present than the A986S variant in this group of Chinese PHPT patients. The R allele increased the risk of PHPT (odds ratio $=1.134,95 \%$ CI: 1.008 , 1.277 , and $P=0.036$ ). Patients with either the RR or RG genotype had lower blood calcium levels and higher alkaline phosphate levels than patients with the GG genotype. The lumbar BMD T-score was $-2.20(-2.63,-0.32)$ in patients with the GG genotype, and it was significantly lower in patients with the $\mathrm{RR}+\mathrm{RG}$ genotype $(-2.53(-3.70,-1.72) \mathrm{P}=0.036)$. Patients with the $\mathrm{R}$ allele had a significantly higher incidence of hyperplasia $(25.0 \%)$ and carcinomas $(7.1 \%)$ than those with the GG genotype (5.3 and $0 \%$ respectively; $P=0.025)$. The prevalence of osteoporosis and parathyroid carcinomas was higher in Chinese PHPT patients with the R allele.

Conclusion: The R990G polymorphism is most frequently present in the Chinese population and among patients with PHPT. Additional studies in the Chinese population are needed to elaborate the relationship between genetics and PHPT.
\end{abstract}

European Journal of Endocrinology 169 629-638

\section{Introduction}

Primary hyperparathyroidism (PHPT) is characterized by excessive endogenous parathyroid hormone (PTH) secretion primarily due to parathyroid adenomas or hyperplasia and, in rare cases, carcinomas. Interestingly, the clinical manifestation and severity of PHPT vary markedly. The variation in PHPT presentation has been reported to be associated with genetic variation of the vitamin D receptor, estrogen receptor, PTH, and calcium-sensing receptor (CASR) $(1,2,3)$.

CASR is a G-protein-coupled receptor expressed in the membrane of parathyroid chief cells, renal tubular epithelial cells, and many other tissues and cells. CASR plays an important role in the maintenance of calcium homeostasis, by sensing the extracellular calcium concentration, regulating PTH secretion, and decreasing renal tubular calcium reabsorption (4). In addition, CASR can influence intestinal calcium absorption (5) and bone remodeling $(6,7)$. Three clustered singlenucleotide polymorphisms (SNPs) have been described for exon 7 encoding the intracellular domain of CASR. The SNPs are as follows: i) a guanine/thymine substitution at codon 986, which leads to the A986S (Ala986Ser) protein variant; ii) an adenine/guanine substitution at codon 990, which leads to the R990G (Arg990Gly) protein variant; and iii) a cytosine/ guanine substitution at codon 1011 , which leads to the Q1011E (Gln1011Glu) protein variant. The impact of these amino acid changes on the function of CASR is 
not well defined. To date, inconsistent results suggesting that the A968S and R990G polymorphisms are associated with mild changes in plasma calcium, phosphate, PTH, alkaline phosphate (ALP), and urinary calcium concentrations have been reported $(8,9)$. There are very few studies to date that have addressed CASR gene polymorphisms in Chinese patients with PHPT.

Kidney stones, or calcification, are common in PHPT patients, but they do not necessarily occur in every patient. Some studies have attempted to investigate the association between CASR gene variants and urinary calcium excretion. Unfortunately, the results have been inconsistent. In one study, an association was detected between urinary calcium excretion and the R990G variant in patients with nephrolithiasis but without PHPT, which suggests that CASR could be a genetic factor regulating calcium excretion (10). By contrast, another study did not find any association between CASR variants and idiopathic hypercalciuria and calcium nephrolithiasis in a French Canadian patient population (11). Limited studies have been carried out regarding the association of the CASR R990G and A986S polymorphisms with osteoporosis or other pathology in PHPT patients. Furthermore, a congeneric CASR genotype-phenotype study in the Chinese population has not been carried out.

The primary objective of this study was to investigate the distribution of the CASR gene A986S, R990G, and Q1011E polymorphisms in Chinese patients with PHPT compared with healthy controls. The secondary objective was to evaluate whether there was an association between genetic polymorphisms and the clinical symptoms of PHPT with an emphasis on kidney stone formation, osteoporosis, and pathology.

\section{Subjects and methods}

\section{Subjects}

Patients with PHPT were recruited from the endocrinology clinic at the Peking Union Medical College Hospital (PUMCH) from October 1983 to May 2009. A total of 452 Chinese patients with sporadic PHPT were followed up at the PUMCH during that time. Of these, 164 unrelated Chinese PHPT patients (113 females and 51 males) agreed to participate in the study and provided whole-blood and serum samples. The mean age of the participants at the initial presentation was $47 \pm 16$ years (range: 13-82). A PHPT diagnosis was made based on elevated total serum calcium and/or ionized plasma calcium levels in the presence of elevated or inappropriate normal serum PTH levels. The diagnosis was confirmed histologically in 131 patients. None of the patients had a familial history suggestive of PHPT or had characteristics of familial benign hypocalciuric hypercalcemia, multiple endocrine neoplasia syndrome, or hyperparathyroidism-jaw tumor (HPT-JT) syndrome.
Nephrolithiasis was detected in $42.7 \%$ of the patients through imaging (X-ray plain film, CT scanning, or ultrasound imaging). Healthy controls were recruited from the health examination center at PUMCH. Age-matched healthy controls $(n=230 ; 180$ females and 50 males) of Chinese Han nationality were selected using the following criteria: i) no personal or family history of kidney stones; ii) normal serum creatinine, calcium, and phosphate levels; and iii) no use of medication that could impact bone metabolism.

This study conformed to the Declaration of Helsinki and was approved by the local ethics committee. All the participants were required to provide informed consent prior to participating in the study.

\section{Laboratory tests}

Fasting venous blood samples were obtained from all the patients to assess the biochemical markers of PHPT. The participants did not have any dietary restrictions. Serum intact PTH, total calcium, phosphate, ALP, and creatinine concentrations were measured at the PUMCH central clinical laboratory using standard methods. Heparinized blood samples were used to measure plasma ionized calcium concentration using the potentiometric method (Bayer $634 \mathrm{PHC} / \mathrm{Ca}^{2+}$ ) within $30 \mathrm{~min}$ of blood collection. Serum intact PTH concentration was measured using chemiluminoimmunoassay (Siemens Immulite 2000, Marburg, Germany). The intra- and inter-assay variability was $<5.0$ and $5.5 \%$ respectively. Urinary calcium and phosphate concentrations were measured using 24-h urine samples. An ultrasound examination of the urinary tract was carried out in all the patients. The bone mineral density (BMD) of the lumbar spine and the proximal femur was measured using dual-energy X-ray absorptiometry.

\section{Genotyping of CASR gene A986S, R990G, and Q1011E polymorphisms}

Blood genomic DNA was extracted by means of standard procedures. The CASR gene was amplified by PCRs. PCRs were carried out using a $50 \mu \mathrm{l}$ reaction mixture containing $0.5 \mu \mathrm{g}$ DNA, $25 \mathrm{pmol}$ of each primer, and 2.5U Taq DNA polymerase AmpliTaq (Quan Shi Jin Company, Beijing, China). The reaction mixtures were amplified under the following cycles: initial denaturation at $94{ }^{\circ} \mathrm{C}$ for $5 \mathrm{~min}$, followed by 30 cycles at $95^{\circ} \mathrm{C}$ for $1 \mathrm{~min}, 58{ }^{\circ} \mathrm{C}$ for $1 \mathrm{~min}, 72{ }^{\circ} \mathrm{C}$ for $1 \mathrm{~min}$, and a final extension step at $72{ }^{\circ} \mathrm{C}$ for $10 \mathrm{~min}$. The primers

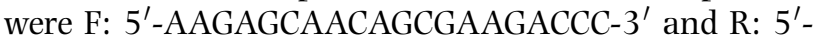
CTCCACCACTGATGACAAAGC- $3^{\prime}$. Direct sequencing of the PCR products was carried out using the Taq BigDye Terminator Sequencing Kit and an ABI3730 automated sequencer (Applied Biosystems). Sequencing was carried out in duplicate. Both the sense and anti-sense strands were sequenced. Sequences generated from the patients were compared with the published CASR 
Table 1 Clinical characteristics of the enrolled patients and the general PHPT patient populations. There was no significant difference in the pathological, clinical, and biochemical characteristics between the 164 recruited and total PHPT patients followed up.

\begin{tabular}{lccc}
\hline & Recruited $(n=164)$ & Total $(n=452)$ & $\boldsymbol{P}$ \\
\hline Serum Ca $(\mathrm{mg} / \mathrm{dl})$ & $11.8 \pm 1.26$ & $11.73 \pm 1.40$ & 0.660 \\
Serum iCa (mmol/l) & $1.82 \pm 0.44$ & $1.50 \pm 0.23$ & 0.975 \\
Serum P (mg/dl) & $2.28 \pm 0.60$ & $2.28 \pm 0.61$ & 0.882 \\
Serum ALP (U/l) & $165(97.33,625.57)$ & $248.65(108.83,886.73)$ & 0.071 \\
Serum PTH (folds of the upper limit) & $7.34(3.87,20.84)$ & $9.04(3.92,19.74)$ & 0.888 \\
Urinary calcium (mmol) & $9.69 \pm 5.67$ & $9.90 \pm 5.81$ & 0.304 \\
Urinary phosphate (mmol) & $18.25 \pm 12.92$ & $20.82 \pm 15.41$ & 0.628 \\
Kidney stones (\%) & 42.68 & 41.9 & 0.926 \\
Bone resorption/osteomalacia & $84 / 143(58.7 \%)$ & $272 / 417(65.2 \%)$ & 0.19 \\
Pathology & & & 0.736 \\
Carcinoma & $8 / 131(6.1 \%)$ & $23 / 395(5.8 \%)$ & \\
Adenoma & $95 / 131(71.8 \%)$ & $290 / 395(73.4 \%)$ & \\
Hyperplasia & $30 / 131(22.1 \%)$ & $82 / 395(20.8 \%)$ & \\
\hline
\end{tabular}

sequence (NCBI reference: A986S: rs1801725; R990G: rs1042636; and Q1011E: rs1801726).

\section{Statistical analyses}

Linkage and Hardy-Weinberg disequilibria were assessed using the Genetic Data Analysis program (computer program for the analysis of allelic data). Quantitative data with a normal distribution are reported as means \pm s.D. For normally distributed data, the Student's $t$-test was used to compare the quantitative difference between the two groups. Data that were not normally distributed are reported as medians and interquartile ranges (25th percentile and 75 th percentile). Nonparametric tests were used to analyze non-normally distributed data. The $\chi^{2}$ test was used to evaluate whether there were significant differences in the frequencies of allele genotypes between the patients and controls, patients with or without kidney stones or calcification, and patients with different pathological results. A binary logistic regression analysis was carried out to determine whether gender and/or gene polymorphisms could predict the occurrence of nephrolithiasis and osteoporosis. Spearman's correlation analysis was used to evaluate whether an association existed between genotypes and the biochemical parameters, nephrolithiasis, and BMD. The unadjusted odds ratios (ORs) for the presence of the R990G and A986S genotypes in PHPT patients and controls were calculated. A 95\% CI was calculated for the summary OR. In addition, we applied a logistic regression approach as implemented in the R package SNPassoc (12) to determine the genotype and phenotype association, and significant associations were assessed using Bonferroni $P$ value correction.

Two-tailed $P$ value $<0.05$ was considered statistically significant. $P<0.025$ and $P<0.016$ were considered statistically significant when determining the association between the allele and haplotype and phenotype after Bonferroni $P$ value correction. All analyses were carried out using the SPSS version 13.0 statistical package and R package SNPassoc (12).

Table 2 SNP frequencies of the R990G and A986S variants in PHPT patients and healthy controls.

\begin{tabular}{lcccc}
\hline & & \multicolumn{3}{c}{ PHPT patients } \\
\cline { 3 - 4 } Allele or haplotype & Controls $(n=230)$ & Total $(n=164)$ & With stones $(n=70)$ & Without stones $(n=94)$ \\
\hline 990 & & & & \\
G & $241(52.4 \%)$ & $147(44.8 \%)$ & $60(42.9 \%)$ & $87(46.3 \%)$ \\
R & $219(47.6 \%)$ & $181(55.2 \%)^{\star}$ & $80(57.1 \%)$ & $101(53.7 \%)$ \\
GG & $61(26.5 \%)$ & $27(16.5 \%)$ & $10(14.3 \%)$ & $17(18.1 \%)$ \\
RR & $50(21.7 \%)$ & $44(21.7 \%)$ & $20(28.6 \%)$ & $24(25.5 \%)$ \\
GR & $119(51.8 \%)$ & $93(51.8 \%)$ & $40(57.1 \%)$ & $53(56.4 \%)$ \\
RR+GR & $169(73.5 \%)$ & $137(83.5 \%)^{\dagger}$ & $60(85.7 \%)$ & $77(81.9 \%)$ \\
986 & & & & $182(96.8 \%)$ \\
A & $445(96.7 \%)$ & $314(95.7 \%)$ & $133(95 \%)$ & $6(3.2 \%)$ \\
S & $15(3.3 \%)$ & $14(4.3 \%)$ & $7(5 \%)$ & $88(93.6 \%)$ \\
AA & $215(93.5 \%)$ & $151(92.1 \%)$ & $64(91.4 \%)$ & $6(6.4 \%)$ \\
AS & $15(6.5 \%)$ & $12(7.3 \%)$ & $5(7.1 \%)$ & 0 \\
SS & 0 & $1(0.6 \%)$ & $1(1.5 \%)$ & $6(6.4 \%)$ \\
SS+AS & $15(6.5 \%)$ & $12(7.9 \%)$ & $6(8.6 \%)$ & \\
\hline
\end{tabular}

${ }^{*} P=0.036, \mathrm{OR}=1.134,95 \% \mathrm{Cl}: 1.008-1.277 ;{ }^{\dagger} P=0.02, \mathrm{OR}=1.255,95 \% \mathrm{Cl}: 1.057-1.490$. 


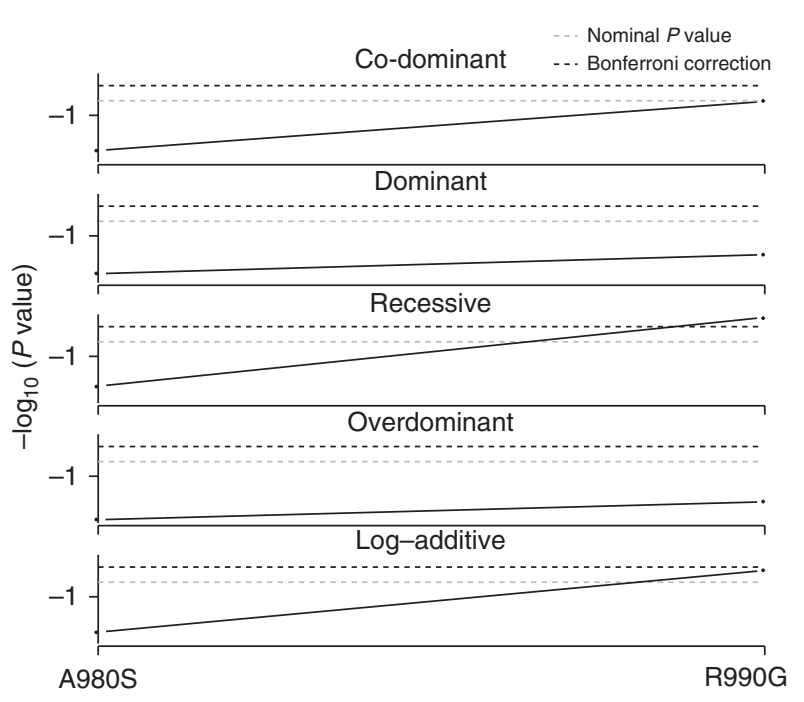

Figure 1 Results of the association between genotypes and PHPT for the case-control study. A logistic regression approach was applied as implemented in the R package SNPassoc to determine the genotype and phenotype association, and significant associations were assessed using Bonferroni $P$ value correction. The $-\log P$ values for the A980S and R990G variants with putative associations are shown for each of the genetic modes. The R990G variant appears to have a significant association with PHPT based on a recessive genetic model $(P=0.017)$.

\section{Results}

To establish that the patient cohort sampled for this study reflects the general PHPT population in China, the biochemical markers and pathology of the enrolled patients $(n=164)$ were compared with those of the total patients $(n=452)$ followed up by the PUMCH. In the 164 enrolled patients selected from the total patients $(n=452)$, the average serum calcium and ionized calcium concentrations were $11.8 \pm 1.26 \mathrm{mg} / \mathrm{dl}$ and $1.82 \pm 0.44 \mathrm{mmol} / \mathrm{l}$ respectively. The average serum phosphate concentration was $2.28 \pm 0.60 \mathrm{mg} / \mathrm{dl}$ and the median PTH level was 7.34 (IQ range: 3.87-20.84) folds of the upper limit. Of the enrolled patients with PHPT, 131 were treated surgically. Of these, PHPT in 95 patients $(71.8 \%)$ was due to adenomas, in 30 patients (22.1\%) due to hyperplasia, and in eight patients $(6.1 \%)$ due to carcinomas. During the same time period, of the 452 Chinese patients with sporadic PHPT followed up by our department, 395 patients had undergone parathyroidectomy. Postoperative pathological findings demonstrated that PHPT in 290 patients $(73.4 \%)$ was due to adenomas, in 82 patients $(20.8 \%)$ due to hyperplasia, and in 23 patients $(5.8 \%)$ due to carcinomas. Overall, no significant differences in the pathological, clinical, or biochemical characteristics of the enrolled patients compared with the general population were observed (Table 1).

\section{Frequency of CASR polymorphisms}

The genotype frequencies of the two CASR gene loci A986S and R990G are given in Table 2. The genotype distribution was consistent with the Hardy-Weinberg equilibrium in both the controls and PHPT patients. In both the PHPT patients and controls, the R990G variant was more frequently present than the A986S variant. Within the R990G variant-containing population, the frequency of the homozygous $R R$ genotype was $16.3 \%$, that of the heterozygous RG genotype was $57.0 \%$, and that of the homozygous GG genotype was $26.7 \%$ for the PHPT patients. In the control population, the frequency of the homozygous RR genotype was $26.5 \%$, that of the heterozygous RG genotype was $51.8 \%$, and that of the homozygous GG genotype was $21.7 \%$. The combined frequency of the RR and RG genotypes was significantly higher in the PHPT patients than in the controls $(\mathrm{OR}=1.259,95 \% \mathrm{CI}$ : $1.060-1.459$, and $P=0.017$ ). Similarly, the $\mathrm{R}$ allele was more frequently present in the PHPT patients than in the controls $(\mathrm{OR}=1.134,95 \%$ CI: $1.008-1.277$, and $P=0.036$ ). Our association analysis showed that the R990G variant might be significantly associated with PHPT based on a recessive genetic model $(P=0.017)$ after Bonferroni correction (Fig. 1 and Table 3).

Within the A986S variant-containing population, the frequencies of each genotype in the PHPT patients and controls were compared. In PHPT patients with the A986S allele, 92.1\% were AA homozygotes. The frequency of the heterozygous AS genotype was 7.3\% and that of the homozygous SS genotype was $0.6 \%$. In the healthy controls, the frequency of the homozygous AA genotype was $93.5 \%$ and that of the heterozygous AS genotype was $6.5 \%$, and no controls homozygous for the SS genotype were detected. There were no significant differences in the genotype and allele frequencies of the A986S variant between the controls and PHPT patients.

Only one PHPT patient was found to have the Gln1011Glu (QE) genotype. All the other patients had

Table 3 Summary of the $P$ values for the case-control study for an association between genotypes and PHPT.

\begin{tabular}{lccccc}
\hline & Co-dominant & Dominant & Recessive & Overdominant & Log-additive \\
\hline A980S & 0.533 & 0.594 & 0.416 & 0.759 & 0.533 \\
R990G & 0.051 & 0.244 & 0.017 & 0.329 & 0.029 \\
\hline
\end{tabular}

The $-\log P$ values for the A980S and R990G variants with putative associations are given for each of the genetic modes. 
the Gln1011 (QQ) genotype. Therefore, owing to the extremely low frequency of variation, the Q1011E SNP was not analyzed further.

\section{SNP haplotype analysis in PHPT}

Further haplotype analysis was carried out to determine the combined effect of these two SNPs on the clinical characteristics of PHPT. Compared with that in patients with the Ala986, Arg990 homozygotes (AR/AR), in patients with the SR/AR or SR/SR genotype, the prevalence of PHPT was low $(\mathrm{OR}=0.299,95 \%$ CI: $0.099-0.908$, and $P=0.028$ ), and in patients with the AG/AR or AG/AG genotype, the prevalence of PHPT was also low $(\mathrm{OR}=0.558,95 \%$ CI: $0.334-0.933$, and $P=0.025)$. However, the difference was not significant after Bonferroni $P$ value correction $(P<0.016)$.

\section{Association between genotypes and phenotypes in PHPT}

Biochemical markers of PHPT In light of the correlation between the CASR R990G polymorphism and PHPT, the association between the clinical characteristics of PHPT and the different genotypes was analyzed. The results are given in Table 4 . After adjusting for serum creatinine concentration and gender, total serum calcium $(P=0.019)$ and plasma ionized calcium $(P=0.01)$ concentrations were significantly higher in patients with the GG genotype than in those with either the RR or RG genotype. However, the elevation of ALP levels was significantly less in patients with the GG genotype compared with that in patients with the $R R+R G$ genotype $(P=0.04)$. No significant differences were observed in the serum phosphate, PTH, and urinary calcium excretion scores between patients with the $\mathrm{R}$ allele (combined genotypes $\mathrm{RR}+\mathrm{RG}$ ) and those with a homozygous $\mathrm{G}$ allele. However, Spearman's correlation analysis did indicate that the R990G variant was associated with serum PTH levels $(r=-0.19$ and $P=0.002)$ and serum phosphate levels $(r=0.294$ and $P=0.002)$. No associations were detected between the measured clinical characteristics and the genotypes of A986S.

Skeletal involvement and BMD To investigate the relationship between CASR polymorphisms and BMD in PHPT patients, we compared the BMD of the lumbar spine and that of the hip in patients with different genotypes. Patients with the $R$ allele $(R R+R G)$ had a significantly lower $T$-score for the BMD of the lumbar spine (L2-L4) than those homozygous for the GG allele (median: -2.53 (IQ range: $-3.70,-1.72$ ) vs median: -2.20 (IQ range: $-2.63,-0.32$ ); $P=0.036$ ). The T-score for the BMD of the hip also tended to be low in patients with the $\mathrm{R}$ allele, although the difference was not significant (Table 5). Patients with the A986S

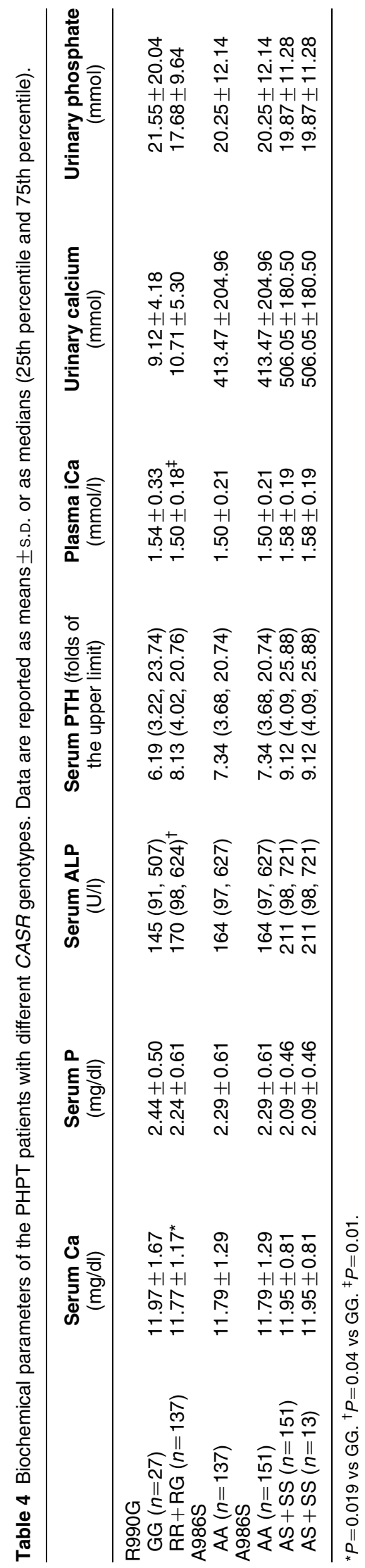

www.eje-online.org 
Table 5 Comparison of the bone mineral density ( $T$-score) in PHPT patients with different calcium-sensing receptor (CASR) genotypes. The T-score of the lumbar (L2-L4) BMD was significantly lower in the patients with the $R R+R G$ genotype than in those with the GG genotype. The data are reported as medians (25th percentile and 75 th percentile). The number of patients used in each analysis is reported $(n)$.

\begin{tabular}{lccc}
\hline Allele & L2-L4 & Neck & Troch \\
\hline R990G & & & \\
GG & $-2.20(-2.63,-0.32)$ & $-2.09(-2.42,-1.19)$ & $-1.37(-2.40,-0.93)$ \\
$n$ & 17 & 17 & 16 \\
RR+GR & $-2.53(-3.70,-1.72)^{*}$ & $-2.32(-2.93,-1.57)$ & $-1.69(-2.68,-1.00)$ \\
$n$ & 79 & 78 & 77 \\
A986S & & $-2.23(2.86,-1.45)$ & $-1.61(-2.64,-0.95)$ \\
AA & $-2.37(-3.36,-1.58)$ & 87 & 85 \\
$n$ & $-37.07(-3.91,-2.08)$ & $-2.30(-2.68,-1.76)$ & $-1.48(-1.79,-0.86)$ \\
AS+SS & 6 & 6 & 6 \\
$n$ & &
\end{tabular}

${ }^{\star} P=0.036$.

genotypes did not have significantly different T-scores for the BMD of either site. No significant differences were found for the incidence of subperiosteal resorption in the phalanges of the hands, dental alveoli and skull, and bone cysts in patients with different genotypes of R990G and A986S.

Factors influencing kidney stone formation To investigate the factors that could be influencing kidney stone formation/calcification, we compared the clinical characteristics of patients with and without kidney stones/calcification. Patients who had kidney stones/ calcification had significantly higher urinary phosphate $(20.81 \pm 8.22$ vs $19.72 \pm 14.24, P=0.049)$ and marginally higher calcium $(11.47 \pm 5.65$ vs $9.85 \pm 5.65$, $P=0.053$ ) excretion than those without kidney stones/ calcification. Patients without kidney stones/calcification had higher serum phosphate $(2.35 \pm 0.67$ vs $2.18 \pm 0.46, P=0.049)$ and PTH $(10.62$ (3.74, 24.6) vs $6.23(4.12,13.59)$ folds of the upper limit, $P=0.059)$ levels than those with nephrolithiasis. Nephrolithiasis was associated with serum PTH levels $(r=-0.172$ and $P=0.034$ ) as shown in Table 6 .

A trend toward a higher prevalence of kidney stone formation/renal calcification was observed in patients with the $\mathrm{R}$ allele ( $\mathrm{RR}+\mathrm{RG}$ genotype) than in those with the GG allele ( 48.3 vs $37.0 \%, P=0.671$ ), but the difference was not significant. To investigate the independent factors contributing to kidney stone formation, a binary stepwise logistic regression analysis was utilized. The exploratory variables were as follows: R990G and A986S variants, gender, serum PTH level, serum calcium concentration, and urinary calcium level. The model indicated that serum PTH levels $(\mathrm{OR}=0.962$, 95\% CI: $0.934-0.990$, and $P=0.008)$ and calciuria $(\mathrm{OR}=1.002$, 95\% CI: 1.000-1.004, and $P=0.027)$ were independent predictors of kidney stone formation.

Pathology Finally, the pathology of patients with the $\mathrm{R}$ allele (combined genotypes $\mathrm{RR}+\mathrm{RG}$ ) and that of the patients with the homozygous GG allele were compared. In patients with the GG genotype, the prevalence of adenomas was significantly higher than that in patients with the RR $+R G$ genotype. The prevalence of hyperplasia and carcinomas was significantly higher in patients with the $R R+R G$ genotype $(P=0.025)$. No significant differences were observed in the pathology of PHPT patients with the AA and AS+SS genotypes (Table 7).

Table 6 Clinical characteristics of PHPT patients with and without nephrolithiasis or calcification. Normally distributed data are reported as means \pm s.D. Intergroup comparisons were made using the Student's $t$-test. Non-normally distributed data are reported as means (25th percentile and 75 th percentile). Nonparametric testing was used to analyze the data.

\begin{tabular}{lccc}
\hline Clinical data & Nephrolithiasis $(n=70)$ & No nephrolithiasis $(n=95)$ & $\boldsymbol{P}$ value \\
\hline Sex (male/female) & $23 / 47$ & $29 / 66$ & 0.493 \\
Age (years) & $47 \pm 14$ & $47 \pm 18$ & 0.551 \\
Serum Ca (mmol/l) & $11.71 \pm 0.91$ & $11.86 \pm 1.46$ & 0.407 \\
Serum PTH (folds of the upper limit) & $6.23(4.12,13.59)$ & $10.62(3.74,24.6)$ & 0.059 \\
Creatinine $(\mu \mathrm{mol} / \mathrm{l})$ & $0.947 \pm 0.33$ & $0.864 \pm 0.41$ & 0.173 \\
Plasma iCa (mmol/l) & $1.50 \pm 0.14$ & $1.51 \pm 0.25$ & 0.957 \\
Serum P (mmol/l) & $2.18 \pm 0.46$ & $2.35 \pm 0.67$ & 0.049 \\
Serum ALP (U/l) & $131(95,275)(n=64)$ & $206(98.53,823.83)(n=88)$ & 0.075 \\
Urinary calcium (mmol) & $11.47 \pm 5.65(n=64)$ & $9.85 \pm 5.65(n=86)$ & 0.053 \\
Urinary phosphate (mmol) & $20.81 \pm 8.22$ & $19.72 \pm 14.24$ & 0.049 \\
GG/RG +RR & $10 / 60$ & $17 / 77$ & 0.671 \\
AA/AS+SS & $64 / 6$ & $88 / 6$ & 0.763 \\
\hline
\end{tabular}


Table 7 Comparison of clinical pathology and kidney stone formation in PHPT patients with different. Significant associations between the R990G genotypes and clinical pathology are shown.

\begin{tabular}{lllll}
\hline \multicolumn{5}{c}{ Pathology } \\
\cline { 2 - 3 } & $\begin{array}{c}\text { Adenomas } \\
(\%)\end{array}$ & $\begin{array}{c}\text { Carcinomas } \\
(\%)\end{array}$ & $\begin{array}{c}\text { Hyperplasia } \\
(\%)\end{array}$ & $\begin{array}{c}\text { Nephrolithiasis } \\
(\%)\end{array}$ \\
\hline R990G & & & & \\
GG & 94.7 & 0 & 5.3 & 37 \\
RR+RG & $67.9^{*}$ & $7.1^{*}$ & $25.0^{*}$ & 43.8 \\
A986S & & & & \\
AA & 70.8 & 6.7 & 22.5 & 42.7 \\
AS+SS & 81.8 & 0 & 18.9 & 46.2 \\
\hline
\end{tabular}

${ }^{*} P=0.025$.

\section{Discussion}

Previously, in the Caucasian population, the A986S variant has been described to be the most frequent polymorphism among the CASR alleles A986S and R990G. The frequency of the A986S allele has been reported to be $12.4 \%$ in west Scotland (13), 16.3\% in Canada (14), 16.7\% in New Zealand (15), and 16.0\% in Sweden (16). By contrast, the results of the present study show that the R990G variant is more frequently present in the Chinese population. Furthermore, the ratio of the $\mathrm{R}$ allele was significantly higher in PHPT patients than in the healthy control population, 55.2 and $47.6 \%$ respectively. The frequency of the A986S allele was only $4.3 \%$ in the PHPT patients and $3.3 \%$ in the healthy controls. The allele frequencies reported herein are consistent with findings in Japanese populations, where the frequency of the R990G allele has been reported to be $40.4 \%$ (8). The Q1011E variant has been reported to be much less frequent than the other two SNPs in the Caucasian population. The frequency of the Q1011E allele has been reported to be only $4.3 \%$ in Canadian postmenopausal women and $2.7 \%$ in Italian postmenopausal women (17). This variant has been shown to be even more infrequent in the Chinese population and detected in only $0.3 \%$ of PHPT patients.

Given that there is a higher prevalence of PHPT in Caucasian populations than in Asian populations $(18,19)$, we hypothesized that differences in genetic factors were contributing to the diversity in disease susceptibility and severity. The present study confirmed that people with the $\mathrm{R}$ allele were more likely to develop PHPT. The frequency of the AR/AR haplotype was higher in this Chinese population, although the difference was not significant. By contrast, Scillitani et al. (3) found that there was a lower frequency of the AR haplotype in Italian PHPT patients than in controls (66.7 vs $71.9 \%$ ). The results suggest that the resulting amino acid changes might decrease the inhibitory activity of the mutated receptor. This would render the parathyroid cells more prone to proliferation and eventually increase the risk of developing PHPT (20). However, two subsequent studies carried out in German and Italian PHPT patients did not detect any positive correlations between the A986S or R990G variants and the risk of PHPT $(9,21)$. The different conclusions drawn from the present study and the previous studies suggest that racial differences may influence the observed associations between genotypes and PHPT.

CASR is expressed in a variety of tissues, including the kidneys and bone. Extracellular calcium ions bind to the extracellular domain of CASR and inhibit the secretion of PTH to maintain serum calcium concentrations within the normal range. The CASR A986S variant has been found to be associated with both total and ionized calcium levels in healthy Caucasian and North American populations $(14,16,21)$. These studies have suggested that CASR polymorphisms may have a significant impact on serum calcium levels. In the present study, we found that blood calcium concentrations were significantly higher in PHPT patients homozygous for the $G$ allele than in those with the $R$ allele $(\mathrm{RR}+\mathrm{RG})$. There was also a trend toward higher serum PTH levels and lower serum phosphate levels in the RR+RG group than in the GG group. These results are similar to those of studies carried out in Japanese PHPT patients (8). Furthermore, the CASR R990G variant has been found to be associated with total calcium and PTH levels in healthy young Han women in Beijing (22). These results support that the CASR R990G variant might have a role in the control of serum calcium stability in the Chinese population.

Osteoporosis is a common manifestation of PHPT. Increasing evidence has suggested that CASR is expressed in stromal cell, preosteoclasts, mature osteoclasts, and various osteoblastic cell lines and may have an important function in bone cells $(7,23$, $24,25,26)$. ALP has been recognized as a bone turnover marker. Consistent with the Japanese studies (8), PHPT patients with the R990 allele had higher ALP levels in the present study. Together, these two studies support that CASR polymorphisms might contribute to bone metabolism in PHPT patients. Furthermore, the $T$-score of the lumbar BMD was significantly lower in patients with the $R$ allele ( $R R+R G$ genotype) than in those with the GG allele. This suggests that patients with the R990 allele might be susceptible to osteoporosis. However, an Italian cohort study did not reveal any correlation between the BMD of the spine or the femur and the prevalence of osteoporosis with R990G genotypes in PHPT patients (20). Another study carried out in postmenopausal Italian women found no significant difference in the frequency distribution of any CASR allele between subjects with and without fragile fracture (17). However, as we have noted, the ethnic difference may contribute to the discrepancy in the conclusions drawn in these studies. Additional ethnic-specific studies are necessary to reach a definite conclusion. 
In Italian populations, the R990G allele has been found to be associated with hypercalciuria in idiopathic calcium stone formers, osteoporotic women, and patients with PHPT $(9,10,27)$. In addition, the R990G SNP has been found to be associated with idiopathic nephrolithiasis in individuals of different ancestries including Canadian, Iranian, and European stone formers and the general population $(28,29,30)$. The same associations have also been found in Italian patients with PHPT $(3,9)$. In the Italian cohort studies, nephrolithiasis has reportedly been shown to be more common in PHPT patients with the RG + GG genotype than in the homozygous RR group, indicating that the R990G allele could be a predisposing factor for nephrolithiasis in PHPT (9). Taking into account that CASR can inhibit tubular calcium reabsorption by acting directly on ion transport (31), the R990G variant could presumably change the sensitivity of CASR to calcium ions (32). In the present study, urinary calcium excretion was higher in patients with the RR + RG genotype than in those with the GG genotype. In addition, the occurrence of kidney stones/calcification tended to be more common in PHPT patients with the RG+RR genotype, but the difference was not significant. The CASR R990G polymorphism was not an independent predictor of nephrolithiasis in this group of Chinese PHPT patients. The divergent results between the present study and previous studies may partly be explained by the different race of the patients. Furthermore, nephrolithiasis was defined only by imaging identification in the present study. In contrast to previous studies, patients who had a history of stone expulsions were not included. Due to the generally low prevalence of PHPT in the Chinese population, the sample size of the present study was limited, especially for the genotype GG carried by only 27 patients. Moreover, numerous factors can affect kidney stone formation, e.g., diet, gastrointestinal disease, and citrate and oxalate concentrations in urine. We did not attempt to include these factors in our analysis, which may have impacted the results.

PHPT results most often from adenomas and somewhat less often from diffuse hyperplasia and, rarely, from parathyroid carcinomas. CASR can be expressed in both normal and malignant cells and may play an important role in the development of various benign and malignant tumors (33). The activation of CASR has an inhibitory effect on the proliferation of parathyroid cells (34). By contrast, inactivating mutations stimulate parathyroid hyperplasia. One possible mechanism whereby CASR could reduce the proliferation of parathyroid cells is through its effects on cyclin D1 (35). Due to the downregulation of the expression of CASR in many parathyroid adenomas, its normal inhibitory function is abrogated, leading to detrimental events that promote the development of parathyroid tumors. Some studies have reported that CASR mRNA is less stable or that one of the CASR alleles on chromosome $3 \mathrm{q}$ is lost in parathyroid adenomas $(36,37)$. A strong downregulation of the expression of
CASR occurs in parathyroid carcinomas with a high proliferative index, a reduction that is even greater than that observed in adenoma and hyperplasia patients, which suggests that CASR probably plays a role in parathyroid cancers (38). This study demonstrated that the incidences of hyperplasia and carcinomas were significantly higher in patients with the $R$ allele ( $R R+R G$ genotype). This could suggest that the amino acid change might reduce the expression of CASR and make the patients with the RG and RR genotypes more likely to develop hyperplasia and carcinomas.

This study explored the association between CASR polymorphisms and pathology of PHPT. However, there are some limitations to the study design. First, no widespread data on the prevalence of PHPT in the general Chinese people were available. The only available data to use as a basis for statistical power calculations were those on the prevalence of PHPT in middle-aged and elderly Chinese males who live in Beijing; therefore, this was not calculated for the present study. Second, due to the study duration (October 1983-May 2009), techniques for the detection of vitamin $\mathrm{D}$ have changed and we were unable to determine vitamin D levels in the study. Third, because of the lower prevalence of PHPT in the Chinese population, the sample size of the study was limited. Further study with a larger sample size is needed to expand and confirm our results.

In conclusion, the R990G allele is the most frequent polymorphism in the Chinese population. People with the $\mathrm{R}$ allele are more likely to develop PHPT. PHPT patients with the GG genotype have higher serum total and ionized calcium levels and lower phosphate levels. Finally, the R990G genotypes might affect the susceptibility to osteoporosis and contribute to the variance in the observed pathology in Chinese PHPT patients.

\section{Declaration of interest}

The authors declare that there is no conflict of interest that could be perceived as prejudicing the impartiality of the research reported.

\section{Funding}

This work was supported by a grant from the National Natural Science Foundation of China (No. 81100559) and National Key Program of Clinical Science.

\section{Acknowledgements}

The authors thank Prof. Wu Shaoyan, working in the department of Biochemistry and Molecular Biology, School of Basic Medical Sciences, Tianjin Medical University, for his help with the statistical analyses.

\section{References}

1 Carling T, Kindmark A, Hellman P, Lundgren E, Ljunghall S, Rastad J, Akerström G \& Melhus H. Vitamin D receptor genotypes in primary hyperparathyroidism. Nature Medicine 19951 1309-1311. (doi:10.1038/nm1295-1309) 
2 Carling T, Rastad J, Kindmark A, Lundgren E, Ljunghall S \& Akerström G. Estrogen receptor gene polymorphisms in postmenopausal primary hyperparathyroidism. Surgery 1997122 1101-1105. (doi:10.1016/S0039-6060(97)90214-2)

3 Scillitani A, Guarnieri V, Battista C, De Geronimo S, Muscarella LA, Chiodini I, Cignarelli M, Minisola S, Bertoldo F, Francucci CM et al. Primary hyperparathyroidism and the presence of kidney stones are associated with different haplotypes of the calcium-sensing receptor. Journal of Clinical Endocrinology and Metabolism 200792 277-283. (doi:10.1210/jc.2006-0857)

4 Brown EM. Physiology and pathophysiology of the extracellular calcium-sensing receptor. American Journal of Medicine 1999106 238-253. (doi:10.1016/S0002-9343(98)00418-5)

5 Chattopadhyay N, Cheng I, Rogers K, Riccardi D, Hall A, Diaz R, Hebert SC, Soybel DI \& Brown EM. Identification and localization of extracellular Ca-sensing receptor in rat intestine. American Journal of Physiology. Gastrointestinal and Liver Physiology 1998 274 G122-G130.

6 Yamaguchi T, Chattopadhyay N, Kifor O, Ye C, Vassilev PM, Sanders JL \& Brown EM. Expression of extracellular calciumsensing receptor in human osteoblastic MG-63 cell line. American Journal of Physiology. Cell Physiology 2001280 C382-C393.

7 Kanatani M, Sugimoto T, Kanzawa M, Yano S \& Chihara K. High extracellular calcium inhibits osteoclast-like cell formation by directly acting on the calcium-sensing receptor existing in osteoclast precursor cells. Biochemical and Biophysical Research Communications 1999261 144-148. (doi:10.1006/bbrc.1999. 0932)

8 Yamauchi M, Sugimoto T, Yamaguchi T, Yano S, Kanzawa M, Kobayashi A \& Chihara K. Association of polymorphic alleles of the calcium-sensing receptor gene with the clinical severity of primary hyperparathyroidism. Clinical Endocrinology 200155 373-379. (doi:10.1046/j.1365-2265.2001.01318.x)

9 Corbetta S, Eller-Vainicher C, Filopanti M, Saeli P, Vezzoli G, Arcidiacono T, Loli P, Syren ML, Soldati L, Beck-Peccoz P et al. R990G polymorphism of the calcium-sensing receptor and renal calcium excretion in patients with primary hyperparathyroidism. European Journal of Endocrinology 2006155 687-692. (doi:10. 1530/eje.1.02286)

10 Vezzoli G, Tanini A, Ferrucci L, Soldati L, Bianchin C, Franceschelli F, Malentacchi C, Porfirio B, Adamo D, Terranegra A et al. Influence of calcium-sensing receptor gene on urinary calcium excretion in stone-forming patients. Journal of the American Society of Nephrology 200213 2517-2523. (doi:10.1097/01.ASN.0000030077.72157.D2)

11 Petrucci M, Scott P, Ouimet D, Trouvé ML, Proulx Y, Valiquette L, Guay G \& Bonnardeaux A. Evaluation of the calcium-sensing receptor gene in idiopathic hypercalciuria and calcium nephrolithiasis. Kidney International 200058 38-42. (doi:10.1046/ j.1523-1755.2000.00138.x)

12 González J, Armengol L, Solé X, Guinó E, Mercader J, Estivill X \& Moreno V. SNPassoc: an R package to perform whole genome association studies. Bioinformatics 200723 644-645. (doi:10. 1093/bioinformatics/btm025)

13 Kelly C, Gunn IR, Gaffney D \& Devgun MS. Serum calcium, urine calcium and polymorphisms of the calcium sensing receptor gene. Annals of Clinical Biochemistry 200643 503-506. (doi:10.1258/ 000456306778904632 )

14 Cole DE, Peltekova VD, Rubin LA, Hawker GA, Vieth R, Liew CC, Hwang DM, Evrovski J \& Hendy GN. A986S polymorphism of the calcium-sensing receptor and circulating calcium concentrations. Lancet 1999353 112-115. (doi:10.1016/S0140-6736(98) 06434-4)

15 Young R, Wu F, Van de Water N, Ames R, Gamble G \& Reid IR. Calcium sensing receptor gene A986S polymorphism and responsiveness to calcium supplementation in postmenopausal women. Journal of Clinical Endocrinology and Metabolism $2003 \mathbf{8 8}$ 697-700. (doi:10.1210/jc.2002-020355)

16 Lorentzon M, Lorentzon R, Lerner UH \& Nordström P. Calcium sensing receptor gene polymorphism, circulating calcium concentrations and bone mineral density in healthy adolescent girls. European Journal of Endocrinology $2001 \quad 144$ 257-261. (doi:10.1530/eje.0.1440257)

17 Cetani F, Pardi E, Borsari S, Vignali E, Dipollina G, Braga V, Adami S, Pinchera A \& Marcocci C. Calcium-sensing receptor gene polymorphism is not associated with bone mineral density in Italian postmenopausal women. European Journal of Endocrinology 2003148 603-607. (doi:10.1530/eje.0.1480603)

18 Yan ST, Tian H, Li CL, Zong WY, Shao YH, Zhong WW \& Gong YP. A preliminary survey of primary hyperparathyroidism in middleaged and elderly Beijing Chinese. Zhonghua Nei Ke Za Zhi 200746 651-653.

19 Lindner G, Felber R, Schwarz C, Marti G, Leichtle AB, Fiedler GM, Zimmermann H, Arampatzis S \& Exadaktylos AK. Hypercalcemia in the ED: prevalence, etiology, and outcome. American Journal of Emergency Medicine 201331 657-660. (doi:10.1016/j.ajem. 2012.11.010)

20 Cetani F, Borsari S, Vignali E, Pardi E, Picone A \& Cianferotti L. Calcium-sensing receptor gene polymorphisms in primary hyperparathyroidism. Journal of Endocrinological Investigation 200225 614-619.

21 Cole DE, Vieth R, Trang HM, Wong BY, Hendy GN \& Rubin LA. Association between total serum calcium and the A986S polymorphism of the calcium-sensing receptor gene. Molecular Genetics and Metabolism 200172 168-174. (doi:10.1006/mgme. 2000.3126)

22 Wang O, Meng XW, Xing XP, Xia WB, Li M, Xu L, Zhou XY, Jiao J, $\mathrm{Hu}$ YY \& Liu HC. Association of calcium-sensing receptor gene polymorphism with serum calcium level in healthy young Han women in Beijing. Zhonghua Nei Ke Za Zhi $2007 \mathbf{4 6} 43-47$.

23 Manolagas SC \& Jilka RL. Bone marrow, cytokines, and bone remodeling. Emerging insights into the pathophysiology of osteoporosis. New England Journal of Medicine 1995332 305-311. (doi:10.1056/NEJM199502023320506)

24 Kameda T, Mano H, Yamada Y, Takai H, Amizuka N, Kobori M, Izumi N, Kawashima H, Ozawa H, Ikeda K et al. Calcium-sensing receptor in mature osteoclasts, which are bone resorbing cells. Biochemical and Biophysical Research Communications 1998245 419-422. (doi:10.1006/bbrc.1998.8448)

25 Chang W, Tu C, Chen TH, Komuves L, Oda Y, Pratt SA, Miller S \& Shoback D. Expression and signal transduction of calcium-sensing receptors in cartilage and bone. Endocrinology $1999 \mathbf{1 4 0}$ 5883-5893. (doi:10.1210/en.140.12.5883)

26 Chattopadhyay N, Yano S, Tfelt-Hansen J, Rooney P, Kanuparthi D, Bandyopadhyay S, Ren X, Terwilliger E \& Brown EM. Mitogenic action of calcium-sensing receptor on rat calvarial osteoblasts. Endocrinology 2004145 3451-3462. (doi:10.1210/en.20031127)

27 Vezzoli G, Terranegra A, Arcidiacono T, Biasion R, Coviello D, Syren ML, Paloschi V, Giannini S, Mignogna G, Rubinacci A et al. R990G polymorphism of calcium-sensing receptor does produce a gain-of-function and predispose to primary hypercalciuria. Kidney International 200771 1155-1162. (doi:10.1038/sj.ki.5002156)

28 O'Seaghdha CM, Yang Q, Glazer NL, Leak TS, Dehghan A, Smith AV, Kao WH, Lohman K, Hwang SJ, Johnson AD et al. Common variants in the calcium-sensing receptor gene are associated with total serum calcium levels. Human Molecular Genetics 201019 4296-4303. (doi:10.1093/hmg/ddq342)

29 Hamilton DC, Grover VK, Smith CA \& Cole DE. Heterogeneous disease modeling for Hardy-Weinberg disequilibrium in casecontrol studies: application to renal stones and calcium-sensing receptor polymorphisms. Annals of Human Genetics 200973 176-183. (doi:10.1111/j.1469-1809.2008.00492.x)

30 Shakhssalim N, Kazemi B, Basiri A, Houshmand M, Pakmanesh H, Golestan B, Eilanjegh AF, Kashi AH, Kilani M \& Azadvari M. Association between calcium-sensing receptor gene polymorphisms and recurrent calcium kidney stone disease: a comprehensive gene analysis. Scandinavian Journal of Urology and Nephrology 2010 44 406-412. (doi:10.3109/00365599.2010.497770) 
31 Blankenship KA, Williams JJ, Lawrence MS, McLeish KR, Dean WL \& Arthur JM. The calcium-sensing receptor regulates calcium absorption in MDCK cells by inhibition of PMCA. American Journal of Physiology. Renal Physiology 2001280 F815-F822.

32 Yano S, Sugimoto T, Kanzawa M, Tsukamoto T, Hattori T, Hattori S \& Chihara K. Association of polymorphic alleles of the calciumsensing receptor gene with parathyroid hormone secretion in hemodialysis patients. Nephron 200085 317-323. (doi:10.1159/ 000045681)

33 Saidak Z, Mentaverri R \& Brown EM. The role of the calcium-sensing receptor in the development and progression of cancer. Endocrine Reviews 200930 178-195. (doi:10.1210/er.2008-0041)

34 Yano S, Sugimoto T, Tsukamoto T, Chihara K, Kobayashi A, Kitazawa S, Maeda S \& Kitazawa R. Association of decreased calcium-sensing receptor expression with proliferation of parathyroid cells in secondary hyperparathyroidism. Kidney International 200058 1980-1986. (doi:10.1111/j.1523-1755.2000. 00370.x)

35 Motokura T, Bloom T, Kim HG, Jüppner H, Ruderman JV, Kronenberg HM \& Arnold A. A novel cyclin encoded by a bcl1-linked candidate oncogene. Nature 1991350 512-515. (doi:10.1038/350512a0)
36 Garrett JE, Capuano IV, Hammerland LG, Hung BC, Brown EM, Hebert SC, Nemeth EF \& Fuller F. Molecular cloning and functional expression of human parathyroid calcium receptor cDNAs. Journal of Biological Chemistry $1995 \quad 270$ 12919-12925. (doi:10.1074/jbc.270.21.12919)

37 Thompson DB, Samowitz WS, Odelberg S, Davis RK, Szabo J \& Heath H III. Genetic abnormalities in sporadic parathyroid adenomas: loss of heterozygosity for chromosome 3q markers flanking the calcium receptor locus. Journal of Clinical Endocrinology and Metabolism 199580 3377-3380. (doi:10.1210/ jc. 80.11 .3377$)$

38 Haven CJ, van Puijenbroek M, Karperien M, Fleuren GJ \& Morreau H. Differential expression of the calcium sensing receptor and combined loss of chromosomes $1 \mathrm{q}$ and $11 \mathrm{q}$ in parathyroid carcinoma. Journal of Pathology 2004202 86-94. (doi:10.1002/ path.1489)

Received 25 May 2013

Revised version received 28 July 2013

Accepted 13 August 2013 